\title{
Aspects of Flavour Physics
}

\author{
Ferruccio Feruglio* \\ Dipartimento di Fisica e Astronomia 'G. Galilei', Università di Padova \\ and INFN Sezione di Padova, Via Marzolo 8, I-35131 Padova, Italy \\ E-mail: feruglio@pd.infn.it
}

\begin{abstract}
Two lectures about the flavour puzzle are summarized in these notes. The theoretical efforts to find a satisfactory description of fermion masses and mixing angles are illustrated by means of simple examples. The emphasis is more on the conceptual points rather than on the construction of realistic models. Two main mechanisms to describe Yukawa interactions are discussed: spontaneously broken abelian flavour symmetries as originally proposed by Froggatt and Nielsen and localization of the profiles for the zero modes of fermions in an extra dimension. Starting from the quark sector the results are extended to the lepton sector and to both $\mathrm{SU}(5)$ and $\mathrm{SO}(10)$ grand unified theories.
\end{abstract}

Proceedings of the Corfu Summer Institute 2014 "School and Workshops on Elementary Particle Physics and Gravity",

3-21 September 2014

Corfu, Greece

${ }^{*}$ Speaker. 


\section{Introduction}

The origin of the parameters in the flavour sector of the Standard Model (SM), minimally extended to include massive neutrinos, is one of the most enigmatic questions in particle physics. We have 22 (20 if B-L is conserved) independent low-energy parameters $\mathscr{Y}_{i}$, or Yukawa couplings, and 18 of them have been measured. Of the remaining four parameters, the absolute scale of neutrino masses is constrained in a limited range, the leptonic Dirac CP-violating phase starts to be constrained by global fits while the two possible Majorana phases are still unknown. A considerable effort has been devoted to search for a more economic description, perhaps related to a new principle, such as the gauge principle. Gauge invariance and renormalizability allow to describe strong and electroweak interactions of three copies of fifteen different fermion species in terms of only three parameters. Nothing similar exists so far in the flavour sector and we usually refer to this as the flavour puzzle.

There are different approaches to the flavour puzzle, with many intermediate possibilities. We may take a reductionist perspective: the Yukawa couplings $\mathscr{Y}_{i}$ should be deduced from first principles. We postulate the existence of a fundamental theory from which $\mathscr{Y}_{i}$ can be uniquely determined. Either by proceeding directly from the candidate theory or by appealing to some symmetry or dynamical principle, $\mathscr{Y}_{i}$ are then computed in terms of a small set of input parameters. Probably the most striking fact about this program is that nothing approaching a standard theory of $\mathscr{Y}_{i}$ exists, despite the decades of experimental progress and theoretical efforts. In another approach a major role is played by chance. There are many variants and practical implementations of this strategy. The Yukawa couplings $\mathscr{Y}_{i}$ are typically mapped to a large number of order-one parameters that are considered as irreducible unknowns, like in models with Froggatt-Nielsen abelian flavour symmetries or with fermions living in extra dimensions. Also the simplest version of partial compositeness falls into this class. By scanning the order-one parameters we get probability distributions for masses and mixing angles. Alternatively we start from a fundamental theory, like string theory, which possesses a vast landscape of solutions, with no privileged ground state. The observed Yukawa couplings become environmental quantities and cannot be predicted, like the relative sizes of the solar planetary orbits [1]. We are allowed to ask much less ambitious questions. For instance, if we have knowledge of the statistical distribution of $\mathscr{Y}_{i}$ in an hypothetical multiverse where the laws of physics follow our fundamental theory, we can ask how typical are the Yukawa couplings that we observe. Conversely, barring anthropic selections, we might assume that the observed $\mathscr{Y}_{i}$ are typical and try to deduce information on the statistical distribution of $\mathscr{Y}_{i}$ in the multiverse $[2,3]$.

In these lectures we will start by reviewing the Froggatt-Nielsen proposal for the quark sector and we will see how it can be adapted to the lepton sector and to grand unified theories as well. The pattern of Yukawa couplings that emerges from this construction can be reproduced also within completely different theoretical schemes, such as models with fermions in an extra dimensions or in partial compositeness and we will briefly review these realizations.

\section{Lessons from the quark sector}

A first useful observation is that ratios of charged fermion masses and quark mixing angles 
can be represented by powers of the Cabibbo angle. Using $\lambda=0.22$ we have

$$
\begin{aligned}
& \frac{m_{e}}{m_{\tau}} \approx \lambda^{5.4} \frac{m_{d}}{m_{b}} \approx \lambda^{4.3} \frac{m_{u}}{m_{t}} \approx \lambda^{7.4} \\
& \frac{m_{\mu}}{m_{\tau}} \approx \lambda^{1.9} \frac{m_{s}}{m_{b}} \approx \lambda^{2.3} \frac{m_{c}}{m_{t}} \approx \lambda^{3.6},
\end{aligned}
$$

where all masses have been renormalised at the scale $m_{Z}$. It is well-known that also the elements of the Cabibbo-Kobayashi-Maskawa (CKM) mixing matrix can be expressed in terms of powers of $\lambda$ :

$$
\left|V_{u d}\right| \approx 1 \quad\left|V_{u s}\right| \approx \lambda \quad\left|V_{c b}\right| \approx \lambda^{2} \quad\left|V_{u b}\right| \approx \lambda^{4} \div \lambda^{3}
$$

For comparison, in the lepton sector, where the Pontecorvo-Maki-Nakagawa-Sakata (PMNS) mixing matrix is denoted by $U$, we have all $\left|U_{f i}\right|$ of order one, except for $\left|U_{e 3}\right|$ which is of order $\lambda$. The ratio between the solar and the atmospheric neutrino squared mass differences $\Delta m_{21}^{2} /\left|\Delta m_{31}^{2}\right|$ is of order $\lambda^{2}$. Focussing on the quark sector, in a pioneering work [4] Froggatt and Nielsen observed that all the small dimensionless parameters of the quark sector such as the quark mass ratios and the CKM mixing angles can be interpreted as powers of the breaking parameter of a flavour symmetry. In this case the flavour symmetry group $G_{f}$ is abelian, $G_{f}=U(1)_{F N}$. A scalar field $\varphi$, carrying by convention a negative unit of the abelian charge $F N$, develops a vacuum expectation value (VEV) that can be parametrized as

$$
\lambda=\langle\varphi\rangle / \Lambda_{f}<1 \quad F N(\varphi)=-1 .
$$

Quarks carry non-negative $\mathrm{U}(1)_{F N}$ charges (the case with charges of both signs can be discussed as well)

$$
F N\left(X_{i}\right) \geq 0 \quad\left(X_{i}=q_{i}, u_{i}^{c}, d_{i}^{c}\right) .
$$

Under these assumptions the quark Yukawa couplings $y_{u, d}$ are given by:

$$
y_{u}=F_{u^{c}} Y_{u} F_{q}, \quad y_{d}=F_{d^{c}} Y_{d} F_{q},
$$

where $Y_{u, d}$ are complex matrices with entries of order one, undetermined by the $\mathrm{U}(1)_{F N}$ symmetry, while $F_{X}$ are real diagonal matrices, completely specified in terms of $\lambda$ by the charges $F N\left(X_{i}\right)$ :

$$
F_{X}=\left(\begin{array}{ccc}
\lambda^{F N\left(X_{1}\right)} & 0 & 0 \\
0 & \lambda^{F N\left(X_{2}\right)} & 0 \\
0 & 0 & \lambda^{F N\left(X_{3}\right)}
\end{array}\right) \quad\left(X_{i}=q_{i}, u_{i}^{c}, d_{i}^{c}\right)
$$

The small quark mass ratios and quark mixing angles originate from the hierarchical structure of the matrices $F_{X}$. Indeed, by taking $F N\left(q_{1}\right)>F N\left(q_{2}\right)>F N\left(q_{3}\right) \geq 0$ we get

$$
\left(V_{u, d}\right)_{i j} \approx \frac{F_{q_{i}}}{F_{q_{j}}}<1 \quad(i<j)
$$

for the matrices $V_{u, d}$ defining the CKM mixing matrix $V_{C K M}=V_{u}^{\dagger} V_{d}$. Independently from the specific charge choice, this framework predicts

$$
V_{u d} \approx V_{c s} \approx V_{t b} \approx O(1) \quad V_{u b} \approx V_{t d} \approx V_{u s} \times V_{c b},
$$


the last equality being correct within a factor of two. With $\lambda \approx 0.2$, the correct order of magnitudes of the $V_{C K M}$ matrix elements can be reproduced by choosing, for instance, $F N(q)=(3,2,0)$. The correct order of magnitudes of the quark mass ratios can be reproduced by choosing, for example

$$
F N(q)=(3,2,0) \quad F N\left(u^{c}\right)=(4,2,0) \quad F N\left(d^{c}\right)=(1+r, r, r),
$$

$r$ being a non-negative integer. If there is only one Higgs doublet, then we need $r$ close to 2 to match the ratio $m_{t} / m_{b}$. If two Higgs doublets are present, other choices are possible by varying $\tan \beta=$ $v_{u} / v_{d}$. Several aspects of this class of models have been discussed in refs. [5, 6, 7, 8, 9, 10, 11, 12].

The construction relies on a spontaneously broken abelian flavour symmetry, but the final results (2.6-2.9) are valid in a more general context, where no symmetry is present to start with. A simple example is provided by a model with an extra spatial dimension, compactified on an orbifold $S^{1} / Z_{2}$ to allow for $4 \mathrm{D}$ chiral fermions. The Lagrangian for a $5 \mathrm{D}$ spinor $\Psi(x, y)$ reads:

$$
\begin{aligned}
\mathscr{L} & =i \bar{\Psi} \Gamma^{M} D_{M} \Psi+m \bar{\Psi} \Psi \\
& =i \bar{\Psi} \gamma^{\mu} \partial_{\mu} \Psi-\bar{\Psi} \gamma_{5} \partial_{y} \Psi+m \bar{\Psi} \Psi+\ldots
\end{aligned}
$$

where the mass $m$ should be odd under the $Z_{2}$ parity sending $y$ into $-y$. A possible choice is

$$
m=M \varepsilon(y),
$$

$M$ being a real constant and $\varepsilon(y)$ the periodic sign function. The 5D spinor has left (L) and right (R) chiralities in four dimensions

$$
\Psi=\left(\begin{array}{l}
\Psi_{L} \\
\Psi_{R}
\end{array}\right)
$$

with opposite $Z_{2}$ parities, such that only the even component developes a massless (zero) mode. Choosing, for instance, $\Psi_{L}$ even and $\Psi_{R}$ odd, the equation satisfied by the zero mode of $\Psi_{L}$ is:

$$
\partial_{y} \Psi_{L}^{0}+M \varepsilon(y) \Psi_{L}^{0}=0
$$

The solution has an exponential dependence on $y$

$$
\Psi_{L}^{0}(x, y)=\sqrt{\frac{2 M}{1-e^{-2 M \pi R}}} e^{-M|y|} \psi(x),
$$

where the first factor provides the correct normalization. The zero mode is localized near $y=0(\pi R)$ for $M>0(<0)$. In the limit $M=0$ the zero mode becomes flat in $y$. A formally identical solution holds for the zero mode of $\Psi_{R}$, if we choose $\Psi_{L}$ odd and $\Psi_{R}$ even and we start from a 5D mass term with the opposite sign. If the Higgs field is strictly localized at one of the two branes, for instance the one at $y=0$, the Yukawa interactions will be proportional to a Dirac delta $\delta(y)$ and we can reproduce the same pattern of Yukawa coupling of eq. (2.6) with matrices $F_{X}$ now given by [13]

$$
F_{X_{i}}=\sqrt{\frac{2 \mu_{i}}{1-e^{-2 \mu_{i} \rho}}}
$$

where $\mu_{i}$ and $\rho$ are specified in terms of the bulk quark masses and the geometry of the extra dimension, see table 1 . The suppression factors $F_{X_{i}}$ represent the values that the profiles of the 


\begin{tabular}{|l|c|c|}
\hline ED & $\mu_{i}$ & $\rho$ \\
\hline Flat $\quad[0, \pi R]$ & $M_{i} / \Lambda$ & $\Lambda \pi R$ \\
\hline Warped $\left[R, R^{\prime}\right]$ & $1 / 2-M_{i} R$ & $\log R^{\prime} / R$ \\
\hline
\end{tabular}

Table 1: Parameters $\mu_{i}$ and $\rho$ in models with an extra dimension compactified on an interval. In a flat (warped) metric the Higgs field is localized on the brane $y=0\left(y=R^{\prime}\right)$ and the ultraviolet cut-off is denoted by $\Lambda(1 / R)$. The fermions are described by five-dimensional spinors, with bulk masses $M_{i}$. In the warped case, when the framework is applied to the gauge hierarchy problem [14], $R, R^{\prime}$ are length scales of the order of the inverse Planck mass and the inverse $\mathrm{TeV}$ scale, respectively.

fermion zero modes take at the brane where the Higgs field is localized. On that brane, generic $O(1)$ Yukawa couplings $Y_{u, d}$ with the bulk quark fields are postulated. The role of the FroggattNielsen charges is here played by $\mu_{i}$ and $\rho$ that determine the profiles along the extra dimension of the zero-mode wave functions:

$$
\sqrt{\rho} F_{X_{i}}=\sqrt{\frac{\xi_{i}}{1-e^{-\xi_{i}}}} \approx\left\{\begin{array}{ll}
\sqrt{\xi_{i}} & \xi_{i} \gg 1 \\
1 & \left|\xi_{i}\right| \ll 1 \\
\sqrt{-\xi_{i}} e^{\xi_{i} / 2} & \xi_{i} \ll-1
\end{array} \quad\left(\xi_{i}=2 \mu_{i} \rho\right) .\right.
$$

There is no flavour symmetry: the hierarchical structure of quark masses and mixing angles is dictated by geometry in the compact space.

Similarly, in the partial compositeness scenario [15], light fermions get hierarchical masses from the mixing between an elementary sector and a composite one. As a toy realization of this idea, consider a model where the composite sector contains, for each SM fermion, a pair of heavy fermions allowing a Dirac mass term of the order of the compositeness scale and a mixing term with the SM fields $[16,17]$

$$
\begin{aligned}
\mathscr{L}_{Y}= & -u^{c} \Delta_{u} U-d^{c} \Delta_{u} D-Q^{c} \Delta_{q} q \\
& -U^{c} M_{u} U-D^{c} M_{d} D-Q^{c} M_{q} Q \\
& -U^{c} Y_{u}\left(\tilde{\Phi}^{\dagger} Q\right)-D^{c} Y_{d}\left(\Phi^{\dagger} Q\right)-\left(Q^{c} \tilde{\Phi}\right) \tilde{Y}_{u} U-\left(Q^{c} \Phi\right) \tilde{Y}_{d} D+\text { h.c. }
\end{aligned}
$$

The first line represents the mixing between elementary and composite sector, the second line displays Dirac mass terms for the fermions of the composite sector and the third line shows the Yukawa interactions that, by assumption, are restricted to the composite sector alone and described by strong couplings $Y_{u, d}, \tilde{Y}_{u, d} \geq 1$. By integrating out the composite sector under the assumption $M_{i} \gg v$, we get low-energy Yukawa interactions for the elementary sector whose leading order (LO) terms have the structure given in eq. (2.6) with matrices $F_{X}$ parametrizing the elementarycomposite mixing:

$$
F_{u^{c}}=\Delta_{u} M_{u}^{-1}, \quad F_{d^{c}}=\Delta_{d} M_{d}^{-1}, \quad F_{q}=M_{q}^{-1} \Delta_{q} .
$$

The same pattern arises when matter chiral multiplets $X_{i}$ of the MSSM are coupled to a superconformal sector in some finite energy range $[18,19,20]$, from an ultraviolet (UV) scale $\Lambda$ down to a lower scale $\Lambda_{c}$. Generic $O(1)$ Yukawa couplings $Y_{i j}$ at the scale $\Lambda$

$$
w=X_{i} Y_{i j} X_{j} H+\ldots
$$


can undergo a sizable renormalization induced by the corrections to the Kahler potential. In the superconformal window the chiral multiplet $X_{i}$ can have a large positive anomalous dimension $\gamma_{i}$ and the Kahler potential at the scale $\Lambda_{c}$ becomes

$$
K=\sum_{i} Z_{i}\left(\Lambda_{c}\right) X_{i}^{\dagger} X_{i}+\ldots
$$

where

$$
Z_{i}\left(\Lambda_{c}\right)=Z_{i}(\Lambda)\left(\frac{\Lambda_{c}}{\Lambda}\right)^{-\gamma_{i}} \quad Z_{i}(\Lambda) \approx 1
$$

Moving to a basis of canonical kinetic terms, the Yukawa couplings at the scale $\Lambda_{c}$ are renormalized

$$
Y_{i j}\left(\Lambda_{c}\right)=F_{X_{i}} Y_{i j} F_{X_{j}} \quad F_{X_{i}}=\left(\frac{\Lambda_{c}}{\Lambda}\right)^{\frac{\gamma_{i}}{2}}<1
$$

and we find again the same pattern of eq. (2.6), without imposing any symmetry.

In the previous examples the anarchical pattern of $Y_{u, d}$ may result in strong bounds on the scale of new physics $\Lambda_{N P}$ associated to particles carrying flavour quantum numbers and representing new sources of FCNC and/or CP violation. In the absence of a concrete realisation, it is difficult to estimate reliably the corresponding effects, also because in general the scale of new physics $\Lambda_{N P}$ and the scale of flavour physics $\Lambda_{f}$ are independent from each other. A possibility is offered by a spurion analysis [21], analogous to that prescribed by Minimal Flavour Violation (MFV)[22]. To this purpose we assume that the new degrees of freedom have non-trivial flavour properties and that the flavour-violating effects are completely specified by the same spurions that are responsible for fermion masses and mixing angles. Moreover we assume that the dominant flavour-violating contributions admit an expansion in power series of the spurion fields. We start by noticing that the pattern of eq. (2.6) is compatible with the flavour symmetry $G_{f}=\mathrm{SU}(3)^{3} \times \mathrm{SU}(3)_{\mathrm{H}}{ }^{3}$ with quarks transforming only under $\mathrm{SU}(3)^{3}$ as

$$
q=(3,1,1) \quad u^{c}=(1, \overline{3}, 1) \quad d^{c}=(1,1, \overline{3}) .
$$

The full symmetry $G_{f}$ is explicitly broken by both the matrices $Y_{u, d}$ and $F_{X}$. However it can be formally restored by treating $Y_{u, d}$ and $F_{X}$ as non-dynamical spurion fields possessing suitable transformation properties. To this aim the Yukawa couplings should transform only under the "hidden" group $\mathrm{SU}(3)_{\mathrm{H}}{ }^{3}$ :

$$
Y_{u}=(3, \overline{3}, 1)_{H} \quad Y_{d}=(3,1, \overline{3})_{H} .
$$

The suppression matrices $F_{X}$ are the interface between $\mathrm{SU}(3)_{\mathrm{H}}{ }^{3}$ and $\mathrm{SU}(3)^{3}$, and they are assigned appropriate transformations under both factors to guarantee the invariance of the Yukawa interactions described by eq. (2.6) under $\mathrm{SU}(3)^{3} \times \mathrm{SU}(3)_{\mathrm{H}}{ }^{3}$. The starting point of the spurion analysis is similar to that of MFV. Indeed the maximal flavour symmetry felt by quarks is $\mathrm{SU}(3)^{3}$, as in MFV. However there are more spurions than in MFV, the irreducible ones including now $F_{q}, F_{u^{c}}, F_{d^{c}}, Y_{u}$ and $Y_{d}$. One of the most dangerous effects originates from the effective operator

$$
\frac{1}{\Lambda_{N P}^{2}}\left(\bar{q} F_{q}^{\dagger} \gamma_{\mu} F_{q} q\right)\left(\overline{d^{c}} F_{d^{c}}^{\dagger} \gamma^{\mu} F_{d^{c}} d^{c}\right) \approx \frac{1}{\Lambda_{N P}^{2}\left\langle Y_{d}^{2}\right\rangle} \frac{2 m_{d} m_{s}}{v^{2}}\left(\bar{s} \overline{d^{c}}\right)\left(s^{c} d\right)+\ldots
$$




\begin{tabular}{|c|cc|cc|}
\hline \hline Operator & \multicolumn{2}{|c|}{ Bounds on $\Lambda_{N P}$ in TeV $\left(c_{\mathrm{NP}}=1\right)$} & \multicolumn{2}{|c|}{ Bounds on $c_{\mathrm{NP}}\left(\Lambda_{N P}=1 \mathrm{TeV}\right)$} \\
& $\mathrm{Re}$ & $\mathrm{Im}$ & $\mathrm{Re}$ & $\operatorname{Im}$ \\
\hline$\left(\bar{s}_{L} \gamma^{\mu} d_{L}\right)^{2}$ & $9.8 \times 10^{2}$ & $1.6 \times 10^{4}$ & $9.0 \times 10^{-7}$ & $3.4 \times 10^{-9}$ \\
$\left(\bar{s}_{R} d_{L}\right)\left(\bar{s}_{L} d_{R}\right)$ & $1.8 \times 10^{4}$ & $3.2 \times 10^{5}$ & $6.9 \times 10^{-9}$ & $2.6 \times 10^{-11}$ \\
\hline$\left(\bar{c}_{L} \gamma^{\mu} u_{L}\right)^{2}$ & $1.2 \times 10^{3}$ & $2.9 \times 10^{3}$ & $5.6 \times 10^{-7}$ & $1.0 \times 10^{-7}$ \\
$\left(\bar{c}_{R} u_{L}\right)\left(\bar{c}_{L} u_{R}\right)$ & $6.2 \times 10^{3}$ & $1.5 \times 10^{4}$ & $5.7 \times 10^{-8}$ & $1.1 \times 10^{-8}$ \\
\hline$\left(\bar{b}_{L} \gamma^{\mu} d_{L}\right)^{2}$ & $6.6 \times 10^{2}$ & $9.3 \times 10^{2}$ & $2.3 \times 10^{-6}$ & $1.1 \times 10^{-6}$ \\
$\left(\bar{b}_{R} d_{L}\right)\left(\bar{b}_{L} d_{R}\right)$ & $2.5 \times 10^{3}$ & $3.6 \times 10^{3}$ & $3.9 \times 10^{-7}$ & $1.9 \times 10^{-7}$ \\
\hline$\left(\bar{b}_{L} \gamma^{\mu} s_{L}\right)^{2}$ & $1.4 \times 10^{2}$ & $2.5 \times 10^{2}$ & $5.0 \times 10^{-5}$ & $1.7 \times 10^{-5}$ \\
$\left(\bar{b}_{R} s_{L}\right)\left(\bar{b}_{L} s_{R}\right)$ & $4.8 \times 10^{2}$ & $8.3 \times 10^{2}$ & $8.8 \times 10^{-6}$ & $2.9 \times 10^{-6}$ \\
\hline \hline
\end{tabular}

Table 2: Bounds on dimension-six $\Delta F=2$ operators, from ref. [23, 24]. The overall coefficient of the operators is $c_{N P} / \Lambda_{N P}^{2}$. The operator discussed in the text is the one in the second row.

$\left\langle Y_{d}^{2}\right\rangle$ representing an average $O(1)$ coupling. The contribution of this operator to the CP-violating $\varepsilon_{K}$ parameter is enhanced at the level of both the hadronic matrix element and the QCD corrections and sets one of the most stringent bounds on the scale of new physics $\Lambda_{N P}$, see Table 2. Assuming a generic $O(1)$ phase for the overall coefficient we need

$$
\left\langle Y_{d}\right\rangle \Lambda_{N P}>20 \mathrm{TeV}
$$

not to spoil the SM prediction for $\varepsilon_{K}$. This, together with other constraints, suggests that a fully anarchical pattern in $Y_{u, d}$ is probably not tenable if new flavoured physics is present at the $\mathrm{TeV}$ scale [25].

When such a spurion analysis is applicable, the estimate of eq. (2.26) represents a sort of lower bound on the size of the expected effect and larger contributions are possible [26]. For example in supersymmetric extensions of the $\mathrm{SM}$ with a $\mathrm{U}(1)_{F N}$ flavour symmetry the operator considered in eq. (2.26) receives contributions from box diagrams with squarks/gluino exchange that are typically larger than the one quoted in eq. (2.26). The reason is that in the $\mathrm{U}(1)_{F N}$ case the true flavour symmetry is much weaker than $\mathrm{SU}(3)_{\mathrm{H}}{ }^{3} \times \mathrm{SU}(3)^{3}$ and it allows sizable off-diagonal terms in both LL and RR blocks for the first two generations of the down squark mass matrix. For instance, with the charge assignment of eq. (2.10), the mass insertions $\left(\delta_{12}^{d}\right)_{L L}$ and $\left(\delta_{12}^{d}\right)_{R R}$ are both proportional to $\lambda$ and the operator $\left(\bar{s} \overline{d^{c}}\right)\left(s^{c} d\right)$ has an overall parametric suppression $1 / 16 \pi^{2} \times \lambda^{2} / \Lambda_{N P}^{2}$, milder than the one in eq. (2.26).

\section{From quarks to leptons}

In the lepton sector we have no evidence for strong hierarchies in mixing angles or in neutrino masses. Hierarchy shows up at the level of charged lepton masses. In terms of the suppression factors $F_{X_{i}}$ this means

$$
F_{e_{1}^{c}} \ll F_{e_{2}^{c}} \ll F_{e_{3}^{c}} \quad \text { and } \quad F_{l_{1}} \approx F_{l_{2}} \approx F_{l_{3}}
$$


For example an acceptable set of charges is

$$
F N\left(e^{c}\right)=(4,2,0) \quad F N(l)=(s+t, s, s) \quad(s \geq 0, t=0,1) .
$$

Here we focus on Majorana neutrinos. In the context of a type I see-saw mechanism right-handed neutrinos $v^{c}$ have their own suppression matrices $F_{v^{c}}$. Yukawa couplings $y_{v, e}$ and the mass matrix $M$ of $v^{c}$ read

$$
y_{v}=F_{v^{c}} Y_{v} F_{l}, \quad y_{e}=F_{e^{c}} Y_{e} F_{l}, \quad M=F_{v^{c}} Y_{c} F_{v^{c}} M_{0},
$$

where $Y_{v, e, c}$ are complex matrices with unknown entries of order one and $M_{0}$ is a mass parameter. At low-energy the active neutrino mass matrix $m_{v}$ is given by

$$
m_{v}=-F_{l}\left(Y_{v}^{T} Y_{c}^{-1} Y_{v}\right) F_{l} v^{2} / M_{0}
$$

with no dependence on the suppression matrices $F_{V^{c}}$.

A drastic realization of this picture is the framework of Anarchy [27, 28, 29, 30, 31], which corresponds to the case

$$
F_{l_{1}}=F_{l_{2}}=F_{l_{3}} \text { or } t=0
$$

In the anarchic framework the mass matrix for light neutrinos is

$$
m_{v}=\left(\begin{array}{ccc}
O(1) & O(1) & O(1) \\
O(1) & O(1) & O(1) \\
O(1) & O(1) & O(1)
\end{array}\right) m_{0} \quad m_{0}=\frac{v^{2}}{M_{0}}
$$

with undetermined order-one matrix elements. This implies mixing angles and neutrino mass ratios of $O(1)$, in rough agreement with the data. No special values for these quantities is expected. Indeed, before we knew $\theta_{13}$ from the experiments, Anarchy successfully anticipated values close to the upper bound at the time. Global fits of present data hint at deviations of the atmospheric mixing angle $\theta_{23}$ from $\pi / 4$. Today these indications are still weak, as shown by the instability of the best fit value against different fitting procedures. The persistence of these deviations in future tests would further strengthen the case for Anarchy. Anarchy represents an extreme possibility and milder realization of the relations (3.5) are possible. For instance, in the context of SU(5) grand unified models, with a Froggatt-Nielsen $\mathrm{U}(1)_{F N}$ abelian symmetry, neutrino masses and mixing angles can be reproduced, at the level of order of magnitudes, by several choices of the FN charges for the $\overline{5}$ multiplets hosting the lepton doublets, as shown in table 3. $F N$ charges for fermions in the 10 representations can be suitably chosen so that, by varying the unknown order-one parameters, reasonable distributions for charged lepton mass ratios, quark mass ratios and quark mixing angles are obtained [32, 33, 34, 35, 36]. A naive comparison of the distributions for neutrino masses and mixing angles with data do not appear to favor Anarchy over the other possible charge assignments, as can be seen from fig. 1. I would personally find more appropriate to use the term Anarchy to denote the approaches giving rise to the results (2.6) and (3.3-3.4) where the absence of any special pattern resides in the matrices $Y$, rather than to indicate the special case defined in eq. (3.5).

If this framework also comprises new flavoured particles at the $\mathrm{TeV}$ scale, severe bounds from lepton flavour violation (LFV) apply, under assumptions analogous to those spelled for the quark sector. The irreducible sources of flavour violation in the lepton sector include the matrices $Y_{e}, F_{e^{c}}$ 

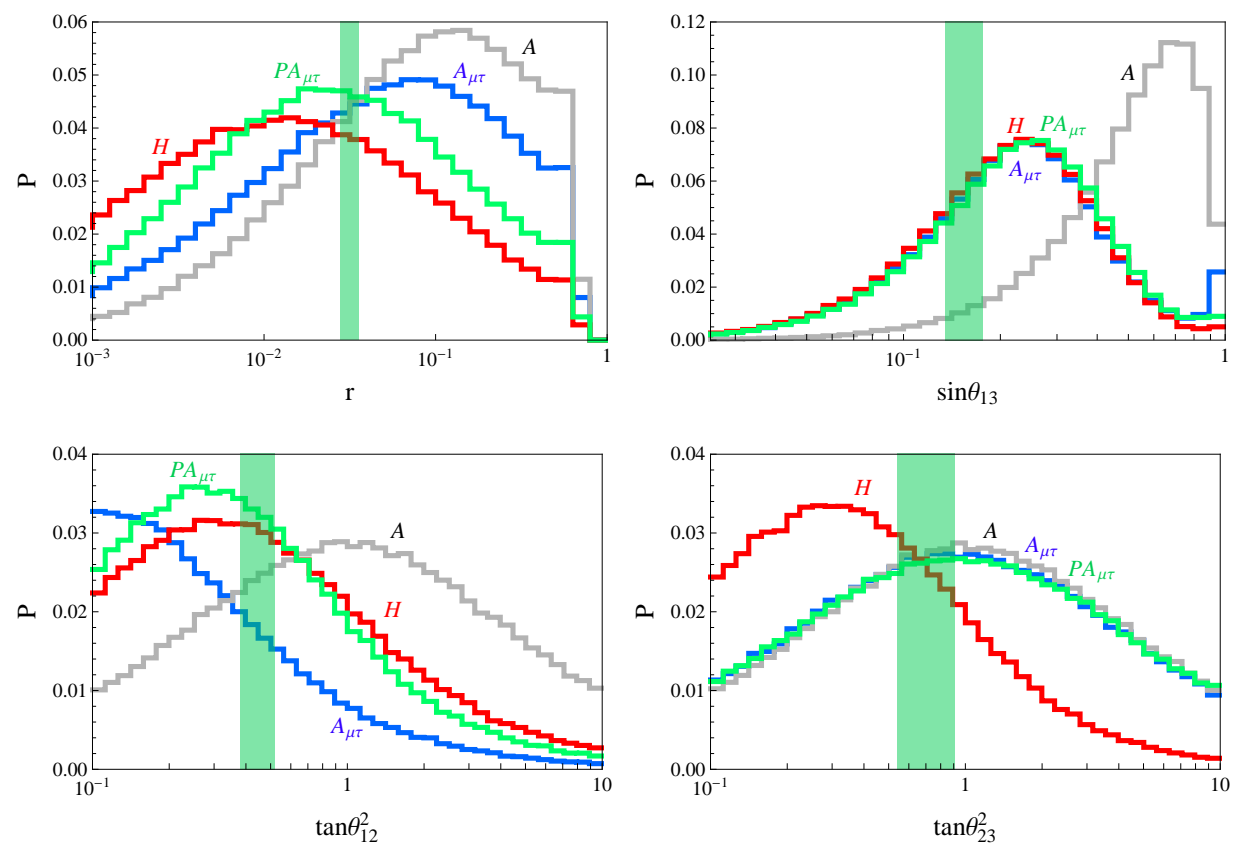

Figure 1: Probability distributions of $r=\Delta m_{s o l}^{2} / \Delta m_{\text {atm }}^{2}, \sin \theta_{13}, \tan ^{2} \theta_{12}, \tan ^{2} \theta_{23}$, from ref. [34], within type I see-saw. The modulus (argument) of the complex random coefficients has been generated in the interval $[0.5,2]([0,2 \pi])$ with a flat distribution. For $A$ and $A_{\mu \tau}, \lambda=0.2$ has been used, for $H$ and $P A_{\mu \tau}, \lambda=0.4$ is taken. The shaded vertical band emphasizes the experimental $2 \sigma$ window.

\begin{tabular}{|l|c|c|}
\hline & $F N(\overline{5})$ & $\lambda$ \\
\hline$A$ & $(0,0,0)$ & - \\
$A_{\mu \tau}$ & $(1,0,0)$ & 0.25 \\
$P A_{\mu \tau}$ & $(2,0,0)$ & 0.35 \\
$H$ & $(2,1,0)$ & 0.45 \\
\hline
\end{tabular}

Table 3: Possible choices of $F N$ charges for the $\overline{5}$ representation in a class of SU(5) grand unified models, from ref. [34]. The second column shows the value of the $F N$ symmetry breaking parameter optimizing the fit to fermion masses and mixing angles.

and $F_{l}$ and LFV can occur even in the limit of vanishing neutrino masses. Notice that, though MFV cannot be extended unambiguously to the lepton sector [37], it predicts no LFV if neutrinos are massless since in this limit the only relevant spurion in the lepton sector is $Y_{e}$, which can always be chosen diagonal. The dipole operator contributing to LFV is

$$
\frac{e}{\Lambda_{N P}^{2}} e^{c} \sigma_{\mu v} F^{\mu v}\left(F_{e^{c}} Y_{e} Y_{e}^{\dagger} Y_{e} F_{l}\right) H^{\dagger} l
$$

The charged lepton mass matrix is proportional to $\left(F_{e^{c}} Y_{e} F_{l}\right)$. In general the combinations $\left(F_{e^{c}} Y_{e} F_{l}\right)$ and $\left(F_{e^{c}} Y_{e} Y_{e}^{\dagger} Y_{e} F_{l}\right)$ are not diagonal in the same basis, not even in the case of universal $F_{l}$ of eq. (3.5), and radiative decays of muon and tau are expected. Agreement with the most constraining upper bound, $B R(\mu \rightarrow e \gamma)<5.7 \times 10^{-13}$, requires $\Lambda_{N P}$ well above $10 \mathrm{TeV}$ [38, 39]. As in the quark sector, a completely anarchical matrix $Y_{e}$ and flavoured physics at the TeV scale are difficult 
to reconcile. A sufficient condition for the absence of LFV is that $Y_{e}, F_{e^{c}}$ and $F_{l}$ are diagonal in the same basis, as suggested in some models. Alternatively we can look for special forms of these matrices $[40,41]$, possibly dictated by some symmetry requirements.

\section{Realizations in grand unified theories}

A welcome feature of the above description is that it can be adapted to grand unified theories (GUT) where quarks and leptons are hosted in the same multiplet of the gauge group. In SU(5) the gauge symmetry requires

$$
F_{q}=F_{u^{c}}=F_{e^{c}}=F_{10} \quad, \quad F_{l}=F_{d^{c}}=F_{\overline{5}}, \quad F_{v^{c}}=F_{1} .
$$

Our previous results, eqs (2.10) and (3.2), come very close to this requirement if we choose $r=s$ and $t=1$. If we accept a couple of tunings in the unknown $O(1)$ parameters $Y_{u, d}$, we can force the equality (4.1) and still have a decent description of both the quark and lepton mass spectrum. As we have seen $F_{v^{c}}$ drops from the low-energy quantities. It is instructive to consider also the ansatz $F_{5} \propto \mathbb{1}$. In this case the hierarchy among fermion masses is entirely due to $F_{10}$. From eqs. $(2.6,3.3)$ we see that mass ratios in the up-quark sector are the square of the respective mass ratios in the down-quark and in the charged lepton sectors, which is correct in first approximation. The large lepton mixing corresponds to a large mixing among $d^{c}$ quarks [42], unobservable in SM weak interactions, but with possible observable effects if transferred from quarks to squarks in SUSY extensions of the SM [43]. A minimal model with Higgs bosons in the 5 representation would lead to the unrealistic relation $y_{e}=y_{d}^{T}$, but the contributions from other Higgs representations or from non-renormalizable operators can solve this problem $[44,45]$ without altering the picture.

At first sight this description does not seem to be compatible with an SO(10) GUT. The most general renormalizable Yukawa interaction of three copies of fermion generations transforming as 16 of $\mathrm{SO}(10)$ reads

$$
\mathscr{L}_{Y}=-\mathbf{1 6}_{i}\left[Y_{10}^{i j} \mathbf{1 0}_{H}+Y_{120}^{i j} \mathbf{1 2 0}_{H}+Y_{126}^{i j} \overline{\mathbf{1 2 6}}_{H}\right] \mathbf{1 6}_{j}+\text { h.c. }
$$

The pattern of Yukawa couplings in eq. (2.6) can also be thought to arise from a rescaling of the fermions fields, with the constraint that fermions belonging to a given irreducible representation of the gauge group have to undergo the same renormalization. By assuming that the matrices $Y_{10}$, $Y_{120}, Y_{126}$ have complex elements of order one and that the fields $\mathbf{1 6}$ undergo a wave function renormalization

$$
16 \rightarrow F_{16} 16,
$$

we see that all members of a $\mathbf{1 6}$ representation are affected in the same way. Even accounting for the Clebsch-Gordan coefficients arising from eq. (4.2) and the different overall scales associated with the Higgs VEVs $\left\langle H_{u, d}\right\rangle$, we cannot reproduce the observed hierarchies of $u, d$ and $e$ masses. Such a discouraging starting point has been successfully modified in a construction by Kitano and Li [46], recently revisited in ref. [47].

The model is a SUSY SO(10) GUT realized in a flat five-dimensional space time, the fifth dimension being compactified on an interval $[0, \pi R]$ whose inverse size is of the order of the GUT scale. The $N=15 \mathrm{D}$ SUSY corresponds to an $N=24 \mathrm{D}$ SUSY, which is broken down to $N=1$ as 
a result of appropriate boundary conditions. Zero modes are allowed only for a vector adjoint supermultiplet $\mathbf{4 5} \mathbf{V}$ and three copies of chiral supermultiplets $\mathbf{1 6}_{\mathbf{i}}$, having bulk masses $M_{i}(i=1,2,3)$. Their $N=2$ superpartners, a chiral adjoint supermultiplet $\mathbf{4 5}_{\phi}$ and conjugate chiral supermultiplets $\mathbf{1 6}^{\mathbf{c}}{ }_{i}$ have only massive modes. A Yukawa superpotential analogous to eq. (4.2) is localized at the brane $y=0$. The key ingredient of the model resides in the gauge interaction of the hypermultiplets. The 5D SUSY gauge interaction contains a 4D Yukawa interaction among $\mathbf{1 6}_{i}, \mathbf{1 6}_{i}{ }_{i}$ and $\mathbf{4 5}_{\phi}$, controlled by the gauge coupling $g_{5}$, that can be combined with the bulk mass term:

$$
-16_{i}^{c}\left[M_{i}-\sqrt{2} g_{5} 45_{\phi}\right] 16_{i}
$$

The chiral multiplet $\mathbf{4 5}_{\phi}$ has no zero mode but can acquire a non-vanishing VEV, $\left\langle\mathbf{4 5}_{\phi}\right\rangle=v_{\Phi}^{3 / 2}$, that breaks $\mathrm{SO}(10)$ down to $\mathrm{SU}(5) \times \mathrm{U}(1)_{X}$. The Yukawa interaction of eq. (4.4) gives rise to effective SO(10)-breaking bulk masses:

$$
\mu_{i}^{r}=\mu_{i}-Q_{X}^{r} k \quad k=\sqrt{2} g_{5} v_{\Phi}^{3 / 2} / \Lambda,
$$

where $\mu_{i}=M_{i} / \Lambda$ and $Q_{X}^{r}$ is the $\mathrm{U}(1)_{X}$ charge of the different $\mathrm{SU}(5)$ components inside the $\mathbf{1 6}$ multiplet: $Q_{X}^{r}=(-1,+3,-5)$ for $r=(10, \overline{5}, 1)$. We are back to the $\mathrm{SU}(5)$ case, see eq. (4.1):

$$
F_{r_{i}}=\sqrt{\frac{2 \mu_{i}^{r}}{1-e^{-2 \mu_{i}^{r} \rho}}} \quad r=(10, \overline{5}, 1)
$$

with $\rho=\Lambda \pi R$, but now the profiles $F_{r_{i}}$ only depend on four free parameters: $\mu_{i}$ and $k$. Neutrinos are described within a type I see-saw mechanism, as in eq. (3.4), with masses for heavy Majorana neutrinos originating from the VEV of the $\mathrm{SU}(5)$ singlet in the $\overline{\mathbf{1 2 6}}_{H}$ representation.

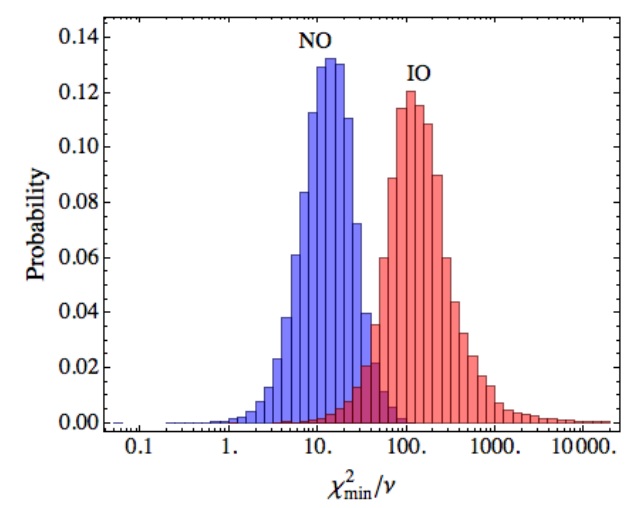

Figure 2: The distributions of minimized $\chi^{2} / v$ for $\mathrm{NO}$ and IO in neutrino masses and for $\tan \beta=50$, from ref. [47].

The model contains many parameters of order one. After rephasing of the relevant fields there are 27 real parameters coming from the matrices $Y_{10}, Y_{120}, Y_{126}$ and 8 real parameters describing the embedding of the two light Higgs doublets within $\mathbf{1 0}_{H}$ and $\mathbf{1 2 0}_{H}$. Despite the large number of parameters the agreement with data is not a priori guaranteed, since there are only 4 profile parameters to describe hierarchical mass ratios and mixing angles. Indeed a fit to an idealized set of 17 observables leads to a good agreement only for large values of $\tan \beta$, for both normal (NO) and inverted (IO) neutrino mass ordering. 

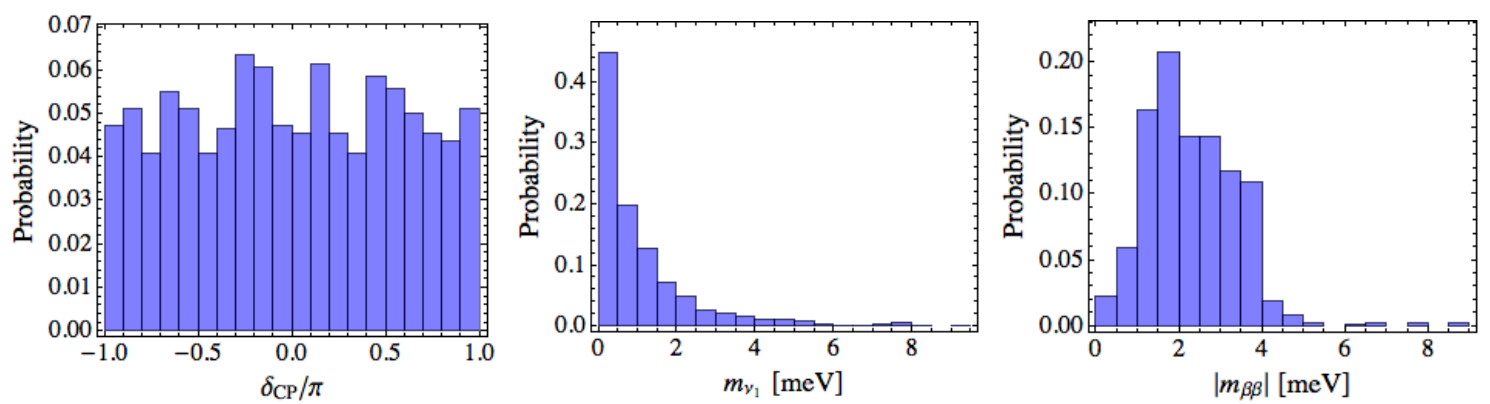

Figure 3: The predictions for various observables obtained for $\chi_{\min }^{2} / \nu<2.21$ in case of NO and $\tan \beta=50$, from ref. [47].

A closer inspection reveals that fitting fermion masses and mixing angles in the IO case requires a fine-tuning of the Yukawa parameters. By generating a large sample of random order-one Yukawa parameters, the fit can be repeated by keeping at each iteration only 12 free parameters, 4 for the profiles and 8 for the relevant Higgs combinations. The distributions of the minimum $\chi^{2}$ over the number of degrees of freedom are shown in fig. 2 for $\mathrm{NO}$ and IO. We see a clear difference between the two cases. While in the IO case we need about $10^{5}$ samples to reach a $p$-value close to 0.05 , in the NO case in about one percent of the cases we have $p>0.05$. The model needs a severe fine-tuning of the "anarchical" parameters in the IO case, while the NO one is realized much more naturally. The most probable values of the profile parameters give $F_{\overline{5}} \approx(0.07,0.22,0.63)$, showing that approximate Anarchy is an output rather than an input of the present construction.

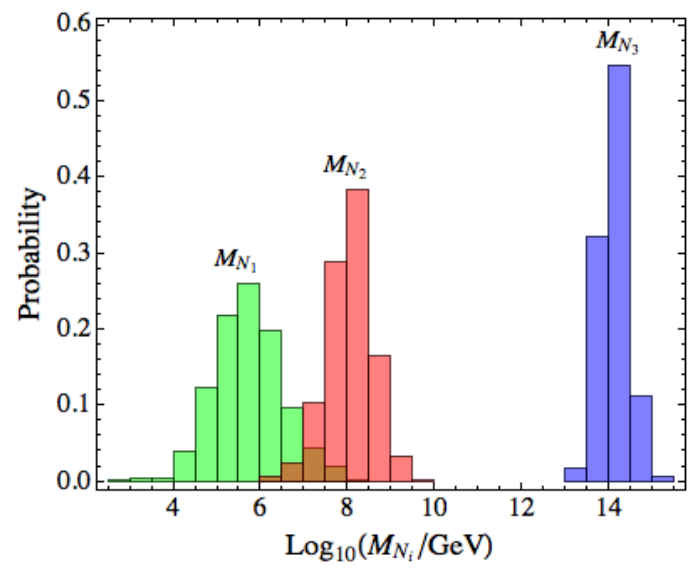

Figure 4: The predictions for the masses of RH neutrinos obtained for $\chi_{\min }^{2} / v<2.21$ in case of NO and $\tan \beta=50$, from ref. [47].

Focussing on the NO case, there is no preferred value of the leptonic Dirac CP phase. The lightest neutrino mass is predicted below $5 \mathrm{meV}$, corresponding to a hierarchical neutrino mass spectrum while $\left|m_{\beta \beta}\right|$ lies in the range $0.1-5 \mathrm{meV}$, see fig. 3. Any positive signal in the current generation of experiments aiming at measuring neutrino masses or $\left|m_{\beta \beta}\right|$ in the lab would essentially rule out the model. The hierarchy in the right handed neutrino spectrum is very pronounced and the corresponding mass distributions are peaked around $10^{6} \mathrm{GeV}, 10^{8} \mathrm{GeV}$ and $10^{14} \mathrm{GeV}$, as shown in fig. 4. 


\section{Conclusion}

In summary, fermion masses and mixing angles are well described by the map in eqs. (2.6,3.3,3.4), in terms of input parameters of order one, the elements of the $Y$ matrices. Such a map can be realized in several different frameworks and does not necessarily need an underlying symmetry. The setup is compatible with both $\mathrm{SU}(5)$ and $\mathrm{SO}(10)$ grand unification and with the known solution to the gauge hierarchy problem. On the weak side, additional ingredients are probably needed to control the new sources of FCNC and CP-violations arising from new flavoured physics at the $\mathrm{TeV}$ scale. Moreover all entries of the $Y$ matrices are independent free parameters and it is not possible to make absolute predictions, beyond the order-of-magnitude accuracy. This is clearly a major limitation, since we would like to test the theory at the level of the best available experimental precision.

\section{Acknowledgments}

FF is very grateful to George Zoupanos and the Organising Committee for inviting him at the Corfu Institute 2014 and for their kind hospitality. The speaker participation and his work were supported in part by the MIUR-PRIN project 2010YJ2NYW and by the European Union network FP7 ITN INVISIBLES (Marie Curie Actions, PITN-GA-2011-289442).

\section{References}

[1] For a review see: A. N. Schellekens, Rev. Mod. Phys. 85 (2013) 4, 1491 [arXiv:1306.5083 [hep-ph]].

[2] J. F. Donoghue, K. Dutta and A. Ross, Phys. Rev. D 73 (2006) 113002 [hep-ph/0511219].

[3] L. J. Hall, M. P. Salem and T. Watari, Phys. Rev. D 76 (2007) 093001 [arXiv:0707.3446 [hep-ph]].

[4] C. D. Froggatt and H. B. Nielsen, Nucl. Phys. B 147, 277 (1979).

[5] M. Leurer, Y. Nir and N. Seiberg, Nucl. Phys. B 398 (1993) 319 [hep-ph/9212278].

[6] M. Leurer, Y. Nir and N. Seiberg, Nucl. Phys. B 420 (1994) 468 [hep-ph/9310320].

[7] L. E. Ibanez and G. G. Ross, Phys. Lett. B 332 (1994) 100 [hep-ph/9403338].

[8] P. Binetruy and P. Ramond, Phys. Lett. B 350 (1995) 49 [hep-ph/9412385].

[9] P. Binetruy, S. Lavignac and P. Ramond, Nucl. Phys. B 477 (1996) 353 [hep-ph/9601243].

[10] N. Irges, S. Lavignac and P. Ramond, Phys. Rev. D 58, 035003 (1998) [hep-ph/9802334].

[11] E. Dudas, S. Pokorski and C. A. Savoy, Phys. Lett. B 356 (1995) 45 [hep-ph/9504292].

[12] E. Dudas, C. Grojean, S. Pokorski and C. A. Savoy, Nucl. Phys. B 481 (1996) 85 [hep-ph/9606383].

[13] D. E. Kaplan and T. M. P. Tait, JHEP 0111 (2001) 051 [hep-ph/0110126]; for early ideas to generate hierarchical Yukawas with extra dimensions see: N. Arkani-Hamed and M. Schmaltz, Phys. Rev. D 61 (2000) 033005 [hep-ph/9903417].

[14] L. Randall and R. Sundrum, Phys. Rev. Lett. 83 (1999) 3370 [hep-ph/9905221].

[15] D. B. Kaplan, Nucl. Phys. B 365 (1991) 259.

[16] R. Contino, T. Kramer, M. Son and R. Sundrum, JHEP 0705 (2007) 074 [hep-ph/0612180]. 
[17] A. J. Buras, C. Grojean, S. Pokorski and R. Ziegler, JHEP 1108 (2011) 028 [arXiv:1105.3725 [hep-ph]].

[18] A. E. Nelson and M. J. Strassler, JHEP 0009 (2000) 030 [hep-ph/0006251].

[19] D. Poland and D. Simmons-Duffin, JHEP 1005 (2010) 079 [arXiv:0910.4585 [hep-ph]].

[20] N. Craig, arXiv:1004.4218 [hep-ph].

[21] K. Agashe, G. Perez and A. Soni, Phys. Rev. D 71 (2005) 016002 [hep-ph/0408134].

[22] G. D’Ambrosio, G. F. Giudice, G. Isidori and A. Strumia, Nucl. Phys. B 645, 155 (2002) [hep-ph/0207036].

[23] G. Isidori, Y. Nir and G. Perez, Ann. Rev. Nucl. Part. Sci. 60 (2010) 355.

[24] G. Isidori, arXiv:1302.0661.

[25] C. Csaki, A. Falkowski and A. Weiler, JHEP 0809 (2008) 008 [arXiv:0804.1954 [hep-ph]].

[26] E. Dudas, G. von Gersdorff, J. Parmentier and S. Pokorski, JHEP 1012, 015 (2010) [arXiv:1007.5208 [hep-ph]].

[27] L. J. Hall, H. Murayama and N. Weiner, Phys. Rev. Lett. 84, 2572 (2000) [hep-ph/9911341].

[28] N. Haba and H. Murayama, Phys. Rev. D 63 (2001) 053010 [hep-ph/0009174].

[29] A. de Gouvea and H. Murayama, Phys. Lett. B 573 (2003) 94 [hep-ph/0301050].

[30] J. R. Espinosa, hep-ph/0306019.

[31] A. de Gouvea and H. Murayama, arXiv:1204.1249 [hep-ph].

[32] G. Altarelli, F. Feruglio and I. Masina, JHEP 0301 (2003) 035 [hep-ph/0210342].

[33] W. Buchmuller, V. Domcke and K. Schmitz, JHEP 1203 (2012) 008 [arXiv:1111.3872 [hep-ph]].

[34] G. Altarelli, F. Feruglio, I. Masina and L. Merlo, JHEP 1211, 139 (2012) [arXiv:1207.0587 [hep-ph]].

[35] G. J. Ding, S. Morisi and J. W. F. Valle, Phys. Rev. D 87 (2013) 5, 053013 [arXiv:1211.6506 [hep-ph]].

[36] J. Bergstrom, D. Meloni and L. Merlo, Phys. Rev. D 89 (2014) 093021 [arXiv:1403.4528 [hep-ph]].

[37] V. Cirigliano, B. Grinstein, G. Isidori and M. B. Wise, Nucl. Phys. B 728 (2005) 121 [hep-ph/0507001].

[38] K. Agashe, A. E. Blechman and F. Petriello, Phys. Rev. D 74 (2006) 053011 [hep-ph/0606021].

[39] C. Csaki, Y. Grossman, P. Tanedo and Y. Tsai, Phys. Rev. D 83 (2011) 073002 [arXiv:1004.2037 [hep-ph]].

[40] M. C. Chen and H. B. Yu, Phys. Lett. B 672 (2009) 253 [arXiv:0804.2503 [hep-ph]].

[41] G. Perez and L. Randall, JHEP 0901 (2009) 077 [arXiv:0805.4652 [hep-ph]].

[42] G. Altarelli and F. Feruglio, Phys. Lett. B 451 (1999) 388 [hep-ph/9812475].

[43] D. Chang, A. Masiero and H. Murayama, Phys. Rev. D 67 (2003) 075013 [hep-ph/0205111].

[44] H. Georgi and C. Jarlskog, Phys. Lett. B 86 (1979) 297.

[45] J. R. Ellis and M. K. Gaillard, Phys. Lett. B 88 (1979) 315.

[46] R. Kitano and T. j. Li, Phys. Rev. D 67 (2003) 116004 [hep-ph/0302073].

[47] F. Feruglio, K. M. Patel and D. Vicino, arXiv:1407.2913 [hep-ph]. 